\title{
100 Gbit/s / 1 V Optical Modulator With Slotted Slow-Light Polymer-Infiltrated Silicon Photonic Crystal
}

\author{
J.-M. Brosi, C. Koos, L. C. Andreani², P. Dumon³, R. Baets ${ }^{3}$, J. Leuthold, and W. Freude \\ Institute of High-Frequency and Quantum Electronics \\ University of Karlsruhe, Engesserstr. 5, 76131 Karlsruhe, Germany \\ ${ }^{2}$ Department of Physics “A. Volta”, University of Pavia, Italy \\ ${ }^{3}$ Ghent University - IMEC, Ghent, Belgium \\ brosi@ihq.uni-karlsruhe.de
}

\begin{abstract}
An optical modulator with $78 \mathrm{GHz}$ bandwidth, $1 \mathrm{~V}$ drive voltage and $80 \mu \mathrm{m}$ length is proposed, allowing $100 \mathrm{Gbit} / \mathrm{s}$ transmission. Design, modulator performance parameters and measurements of the slow-light photonic crystal waveguide are discussed.

(C) 2008 Optical Society of America

OCIS codes: (130.5296) Photonic crystal waveguides; (130.5460) Polymer waveguides; (130.4110) Modulators.
\end{abstract}

\section{Introduction}

Silicon photonic opens a cost-effective route by processing silicon-on-insulator (SOI) wafers with well-established CMOS technology. Rapid progress has been made in developing silicon modulators which are driven by free carrier injection up to $30 \mathrm{GHz}$ [1]. Much faster, but narrow-banded modulation at $165 \mathrm{GHz}$ has been shown with nonlinear electro-optic polymers [2], which can also be employed as cladding material for silicon slot waveguides (WG) [3]. In photonic crystal (PC) WGs, the group velocity of light may be reduced, which significantly enhances nonlinear interaction and enables efficient modulation [4].

We propose a silicon Mach-Zehnder interferometer (MZI) modulator with $78 \mathrm{GHz}$ modulation speed and $1 \mathrm{~V}$ drive voltage [5]. This performance allows $100 \mathrm{Gbit} / \mathrm{s}$ transmission. Our design combines the large field concentration of slot WGs, the high nonlinearity of polymers, and the increased interaction time by a slow-light photonic crystal structure. By careful design, chromatic dispersion can be eliminated in a bandwidth of $1 \mathrm{THz}$. Measurements demonstrate the feasibility of the slow-light structure.

\section{Modulator Design}

The MZI modulator is schematically shown in Fig. 1(a). The input light is split in two arms, where phase modulators (PM) are inserted and driven in push-pull mode. The PM is realized with slot PC-WGs, which are infiltrated with a highly nonlinear polymer. In such WGs, the optical field is mainly confined to the slot, Fig. 1(b). The modulating PM voltage is applied to the electrodes and transferred to the slot region via conductive silicon slabs. Like the optical field, the modulating voltage is essentially confined to the slot, leading to a large electro-optic interaction. The

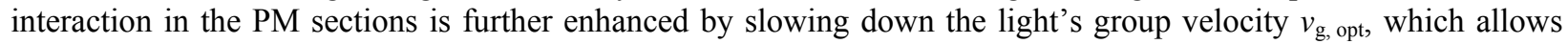
reducing the modulator size. To avoid distortions of optical pulses, the chromatic dispersion has been flattened in a $1 \mathrm{THz}$ bandwidth, see Fig. 1(c). Without dispersion flattening (i. e., all holes have same radii), the group velocity changes strongly with optical frequency (dashed line).
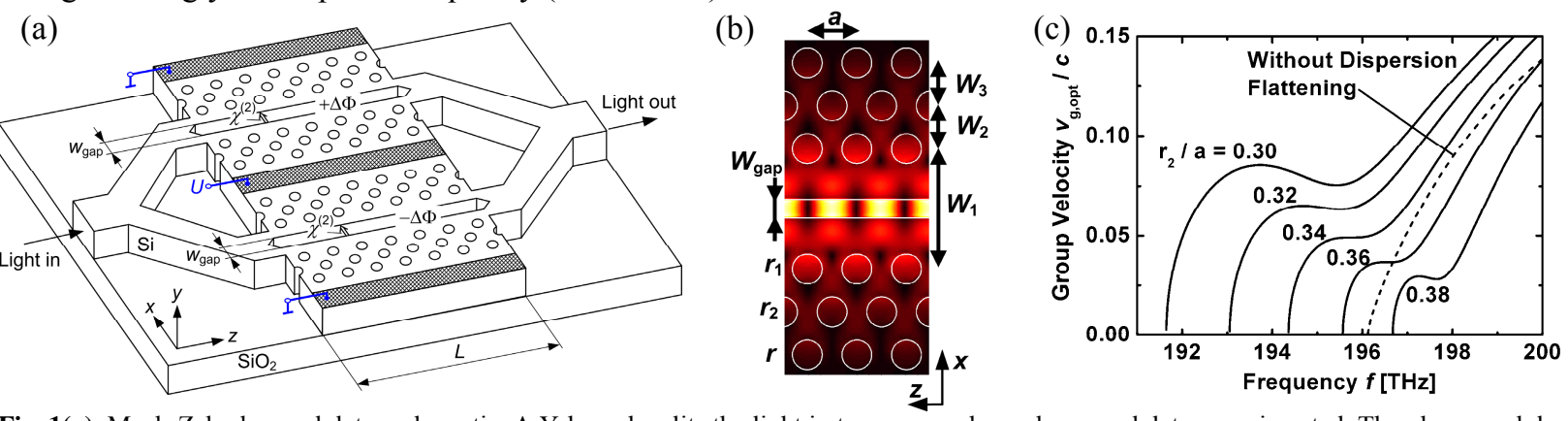

Fig. 1(a). Mach-Zehnder modulator schematic. A Y-branch splits the light in two arms, where phase modulators are inserted. The phase modulation takes place in a narrow gap $w_{\text {gap }}$ filled with a $\chi^{(2)}$-nonlinear polymer inside a PC-WG. The PC is designed to have low group velocity, which enhances the nonlinear interaction. The silicon of the PC is doped. Electrodes (shaded) are attached to supply the modulating voltage $U$. Fig. 1(b). Dominant optical E-field distribution. In the polymer-filled gap, both modulating voltage and optical field are strongly confined. Fig. 1(c). Group velocity of slotted photonic crystal WG. Region of constant low group velocity controlled with $r_{2}$. Parameters: $W_{1}=1.25 \sqrt{ } 3 a$, $W_{2}=0.65 \sqrt{ } 3 a, W_{3}=0.45 \sqrt{ } 3 a, r_{1}=0.25 a, r=0.3 a$, silicon slab height $220 \mathrm{~nm}$, gap width $W_{\text {gap }}=150 \mathrm{~nm}$, PC period $a=408 \mathrm{~nm}$. $(---): v_{\mathrm{g}, \text { opt }}$ without dispersion flattening. 


$$
\begin{gathered}
f_{3 \mathrm{~dB}}=\frac{0.5 v_{\mathrm{g}, \mathrm{ppt}}}{L} \frac{1}{\left|1+v_{\mathrm{g}, \mathrm{ppt}} / v_{\mathrm{g}, \mathrm{el}}\right|} \approx \frac{0.5 v_{\mathrm{g}, \mathrm{opt}}}{L} \\
U_{\pi}=\frac{C}{n_{\mathrm{poly}}^{3} f_{0}} \frac{W_{\text {gap }}}{r_{33}} \frac{1}{\Gamma L} \propto \frac{W_{\text {gap }}}{r_{33}} \frac{v_{\mathrm{g}, \mathrm{ppt}}}{L}
\end{gathered}
$$

Table 1

Characteristic Data for PC Slot Waveguide Modulator

\begin{tabular}{ccccc}
\hline \hline$r_{2} / a$ & $f_{\mathrm{o}}(\mathrm{THz})$ & $v_{\mathrm{g}, \mathrm{opt}} / c$ & $L(\mu \mathrm{m})$ & $f_{3 \mathrm{~dB}}(\mathrm{GHz})$ \\
\hline 0.38 & 197.5 & $3.2 \%$ & 57 & 87 \\
$\mathbf{0 . 3 6}$ & $\mathbf{1 9 6 . 5}$ & $\mathbf{4 . 0 \%}$ & $\mathbf{8 0}$ & $\mathbf{7 8}$ \\
0.34 & 195.8 & $5.2 \%$ & 111 & 71 \\
0.32 & 195.2 & $6.6 \%$ & 158 & 61 \\
0.30 & 194.6 & $7.9 \%$ & 215 & 53
\end{tabular}

In Table 1 and from Eqs. (1)-(2), estimates of the MZI modulation bandwidth $f_{3 \mathrm{~dB}}$ and modulator length $L$ for a fixed phase modulator's $\pi$-voltage $U_{\pi}$ and modulation amplitude $\hat{U}=U_{\pi} / 4=1 \mathrm{~V}$. The electrical group velocity is $v_{\mathrm{g}, \mathrm{el}}$, $n_{\text {poly }}=1.6$ and $r_{33}=80 \mathrm{pm} / \mathrm{V}$ are the refractive index and electro-optic coefficient of the polymer, and $\Gamma$ stands for the electro-optic field interaction factor [5]. The data in Table 1 refer to the dispersion-flattened structures in Fig. 1(c). Out of these structures, one with a sufficiently flat dispersion at a realizable optical group velocity of $4 \%$ of the vacuum speed of light was chosen, bold-face line in Table 1. A large modulation bandwidth of $78 \mathrm{GHz}$ and a short phase modulator length of only $80 \mu \mathrm{m}$ are predicted. The bandwidth $f_{3 \mathrm{~dB}}$ is limited by the walk-off between the optical and electrical signal, while electrical $R C$-effects do not play a role [5].

\section{Waveguide Measurements}

A slot PC-WG, presently without dispersion flattening, has been fabricated using deep-UV lithography. It is covered with a glass cladding. The group velocity characteristic corresponds to the dashed line in Fig. 1(c). The PC slot-WG is fed by strip WGs, schematically shown as inset in Fig. 2(b). For increasing the coupling to the slow-light mode, PC tapers (not shown in inset Fig. 2(b)) are inserted at both sides of the PC-WG. The light was coupled to the quasiTE mode using polarization maintaining lensed fibers. For two different PC lengths the transmitted power relative to the one of a straight strip waveguide is shown in Fig. 2(a). The corresponding group delays were measured by a phase shift method, Fig. 2(b). The oscillations in the transmission and group delay characteristics are caused by reflections at the silicon/air interface. For the long PC-WG, transmission dips can be observed, which we attribute to disorder-induced mode coupling. The minimal transmission loss of the PC slot-WG is about $9.5 \mathrm{~dB} / \mathrm{mm}$ due to fabrication imperfections. This, however, is not an issue because the modulator length is very small, see Table 1 . The thick lines in Fig. 2(b) represent the numerically calculated group delays, which agree well with the experimental data. At $189.5 \mathrm{THz}$ a group velocity of $6 \%$ of the vacuum speed of light can be inferred.

(a)

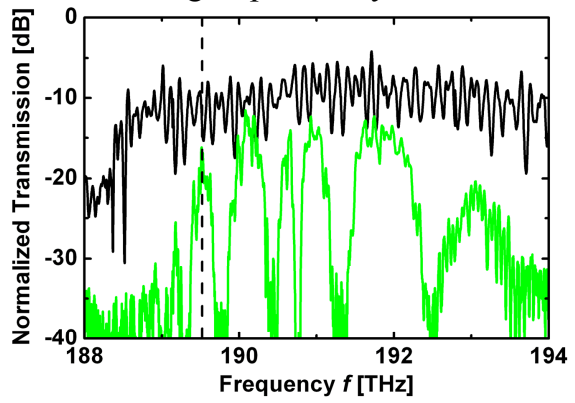

(b)

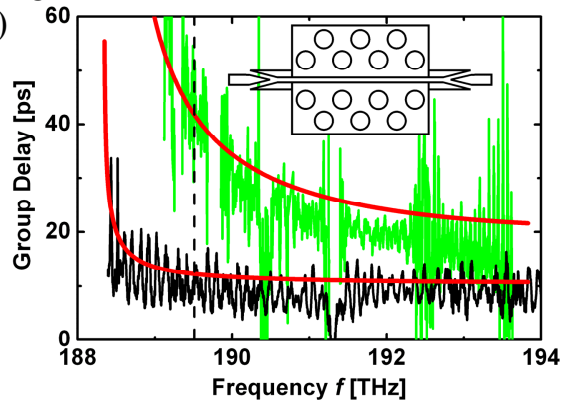

Fig. 2(a). Transmission of PC slot-WGs including coupling structures, see inset Fig. 2(b). The data are normalized to the transmission of strip WGs. The curves correspond to PC lengths of $48 \mu \mathrm{m}$ and $642 \mu \mathrm{m}$. The minimum transmission losses of the PC slot-WG are $9.5 \mathrm{~dB} / \mathrm{mm}$. Fig. 2(b). Group delay of PC slot-WGs. Inset: Device structure with transitions from strip-WG to slot-WG. Calculated delay curves (thick lines) agree well with measurements. At $189.5 \mathrm{THz}$, the group velocity is $6 \%$ of the vacuum speed of light.

Acknowledgement This work was supported by the Deutsche Forschungsgemeinschaft (DFG) in the framework of the Priority Program SP 1113 "Photonic Crystals", and by the Deutsche Telekom Stiftung.

\section{References}

[1] L. Liao, A. Liu, D. Rubin, J. Basak, Y. Chetrit, H. Nguyen, R. Cohen, N. Izhaky, and M. Paniccia, “40 Gbit/s optical modulator for highspeed applications," Electron. Letts., vol. 43, no. 22, Oct. 2007.

[2] B. Bortnik, Y.-C. Hung, H. Tazawa, B.-J. Seo, J. Luo, A. K.-Y. Jen, W. H. Steier, and H. R. Fetterman, "Electrooptic polymer ring resonator modulation up to $165 \mathrm{GHz}$," IEEE J. Sel. Topics Quantum Electron., vol. 13, pp. 104-110, Jan./Feb. 2007.

[3] M. Hochberg, T. Baehr-Jones, G. Wang, J. Huang, P. Sullivan, L. Dalton, and A. Scherer, "Towards a millivolt optical modulator with nanoslot waveguides," Opt. Expr., vol. 15, pp. 8401-8410, Jun. 2007.

[4] L. Gu, W. Jiang, X. Chen, L. Wang, and R. T. Chen, "High speed silicon photonic crystal waveguide modulator for low voltage operation," Appl. Phys. Lett., vol. 90, 071105, Feb. 2007.

[5] J.-M. Brosi, C. Koos, L. C. Andreani, M. Waldow, J. Leuthold, and W. Freude, "High-speed low-voltage electro-optic modulator based on a CMOS compatible polymer-infiltrated silicon photonic crystal," Opt. Expr. (accepted for publication) 